\title{
Relevance of benign prostatic hyperplasia and associated conditions for urologists, health care systems, and society
}

\author{
Matthias Oelke - Stavros Gravas
}

Received: 4 January 2010/Accepted: 7 January 2010/Published online: 14 January 2010

(c) The Author(s) 2010. This article is published with open access at Springerlink.com

The guest editors of this special issue, both members of the European Association of Urology Guidelines Office dealing with male lower urinary tract symptoms and benign prostatic hyperplasia (BPH), have been asked by the editorial board of the World Journal of Urology to collect and publish a series of articles on BPH to document the latest developments of this disease. Next to this issue solely dealing with the treatment of BPH, another special issue on basic science, epidemiology, and assessment of BPH is already in preparation and anticipated to be published later in 2010. Both special issues contain a mixture of original scientific as well as review articles on BPH and summarize the latest knowledge of the BPH disease. We thank all authors who have followed our invitation and shared their professional inside information with us. As all articles have been peer-reviewed, we would also like to thank all reviewers for their valuable time, work, and suggestions.

BPH is the term used only to describe benign growth of epithelial, muscular, and/or fibrotic cells in the prostate. Microscopic changes increase with ageing and approximately $40,70,80$, and $90 \%$ of men are affected in their sixth, seventh, eighth, and ninth decade, respectively [1]. Based on the prevalence data, BPH appears to be a

M. Oelke ( $\square)$

Department of Urology, Hannover Medical School,

Carl-Neuberg-Str. 1, 30625 Hannover, Germany

e-mail: oelke.matthias@mh-hannover.de

M. Oelke

Department of Urology, Academic Medical Center,

University of Amsterdam, Amsterdam, The Netherlands

S. Gravas $(\bowtie)$

Department of Urology, University of Thessaly,

6-8, Feidiou Street, 41221 Larissa, Greece

e-mail:sgravas2002@yahoo.com physiological ageing process. BPH becomes a disease when benign prostatic enlargement (BPE), benign prostatic obstruction (BPO), or lower urinary tract symptoms (LUTS) appear. No strict correlations between these components have been found so far and, therefore, each component of the BPH disease has to be assessed and treated separately. It is estimated that one-third to one-half of men with BPH develop BPE, approximately half of men with BPH-LUTS have BPO, and up to $40 \%$ of men in a community have LUTS.

LUTS is the most frequent cause of annoyance and decreased life quality in patients with BPH and associated with health-seeking behavior [2]. Although in most cases not life-threatening, BPH-LUTS has a tremendous socioeconomic impact. Data from the Integrated Health Care Information Solutions National Managed Care Benchmark Database which included more than 30 health care plans between July 1997 and January 2003 and covered 25 million men aged 50 years or older demonstrated that BPE-LUTS is the fifth most commonly treated disease in the US, following cataracts, hypertension, osteoarthritis, and bursitis [3]. It was calculated from this database that medical costs of BPE-LUTS are among the seven most costly diseases. The chance of developing a significant clinical event (defined as acute urinary retention or need for prostate surgery) within 12 months after initiating medical treatment was the second highest of all diseases. Inpatient treatment of BPE-LUTS was responsible for $40 \%$ of the total costs which were only higher for cataracts, coronary artery diseases, osteoarthritis, and arrhythmias. It was concluded that improvement of the understanding of BPE (BPO or LUTS) would have the potential to significantly decrease costs. However, it is expected that the number of patients will dramatically increase as the average life expectancy increases worldwide, and therefore, further 
improvements in assessment and treatment of BPE, BPO, and LUTS will help in decreasing the cost related to BPHrelated conditions.

Assessment and treatment of BPH, BPE, BPO, or male LUTS has been and will be the "bread and butter" of urologists not only because of the pure amount of patients, but also because of the special skills necessary to treat these patients. Urology would definitely not exist as a separate specialization without $\mathrm{BPH}$ and $\mathrm{BPH}$-specific treatments. Amazingly, the importance of this disease is not reflected by the amount of publications in peerreviewed journals or abstracts of major urology congresses. Of 536 published articles in European Urology in 2009, only 12 articles $(2.2 \%)$ dealt with BPH or male LUTS. Therefore, the number one topic of urology is clearly underrepresented in the current literature. The guest editors of this issue of the World Journal of Urology are therefore grateful to have the opportunity to draw more attention to the most frequent urological disease. We wish our readers a joyful and interesting time reading the following articles on medical or surgical treatment of clinical BPH.

Yours,

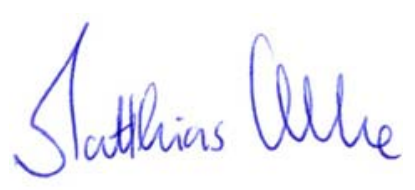

Matthias Oelke

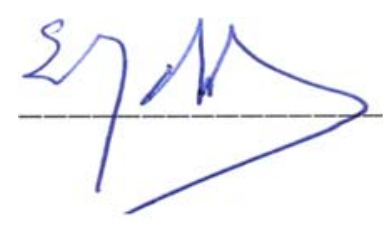

Stavros Gravas
Conflict of interest statement The authors have no conflicts of interest.

Open Access This article is distributed under the terms of the Creative Commons Attribution Noncommercial License which permits any noncommercial use, distribution, and reproduction in any medium, provided the original author(s) and source are credited.

\section{References}

1. Berry SJ, Coffey DS, Walsh PC, Ewing L (1984) The development of human benign prostatic hyperplasia with age. J Urol 132:474-479

2. Hutchison A, Farmer R, Chapple C et al (2006) Characteristics of patients presenting with LUTS/BPH in six European countries. Eur Urol 50:555-562

3. Fenter TC, Naslund MJ, Shah MB et al (2006) The cost of treating the 10 most prevalent diseases in men 50 years of age or older. Am J Manag Care 4(Suppl):S90-S98 